\title{
Design and Analysis of Pulse width Modulator (PWM) using Low Input Impedance Current Comparator
}

\author{
Rockey Choudhary ${ }^{1}$, Prof. B.P. Singh ${ }^{2}$ \\ ${ }^{I}$ (M.Tech(VLSI design) at Mody Institute of Technology \&Science,Laxmangarh (Sikar), India) \\ ${ }^{2}$ (Mody Institute of Technology \&Science, Department of ECE,Laxmangarh (Sikar), India)
}

\begin{abstract}
In this paper a new design of a simple pulse width modulator is presented. The proposed scheme has $3 \mathrm{~mW}$ average power consumption which is less than the existing PWM methods. This circuit is also suitable for IC implementation. The simulation results are carried out under the implementation of TSMC $0.35 \mu \mathrm{m}$ CMOS process with $1.8 \mathrm{~V}$ power supply. This method is commonly used for driving motors, heaters, or lights in varying intensities or speeds. The simulation and experimental results are shown in this paper. It is also suitable for high power applications as well as ordinary low power use, while possessing important advantages. Pulse-width (PW) modulation has been widely used to convert analog (or multibit digital) signals to binary streams.
\end{abstract}

Keywords - Current Comparator, CMOS Integrated circuits, PWM, summer circuit, Triangular wave generator,

\section{INTRODUCTION}

Pulse Width Modulation, is a method of controlling the amount of power to a load without having to dissipate any power in the load driver. The voltage-mode comparator is the most critical component of pulse-width modulator which dominates the accuracy of modulation process. However, voltage-mode comparator encounters several great difficulties including operational frequency, power consumption and input offset voltage. The current-mode comparator therefore has been attracted considerable attention as it is more easily to achieve design requirements than voltage-mode circuits. With respect to application of pulse-width modulation, input impedance of current comparator is the most important specification than operational frequency, power consumption and other specifications. The lower input impedance enhances the transformation efficiency of the signal and increases the accuracy of modulation process as well.

PWM is currently realised in integrated circuits (ICs) form and that make it convenient to utilize. Some simple PWM using relaxation oscillator have been proposed [1,2]. Although its scheme is relatively simpler but its duty factor does not linearly vary with modulating signal.

This paper proposed a simple PWM which can be realised in IC form. Its major advantage is that not only it can generate accuracy PWM signal with widely operating frequency but also its scheme is simpler than conventional PWM IC form. The simulation and experimental results are found to agree well with our anticipation.

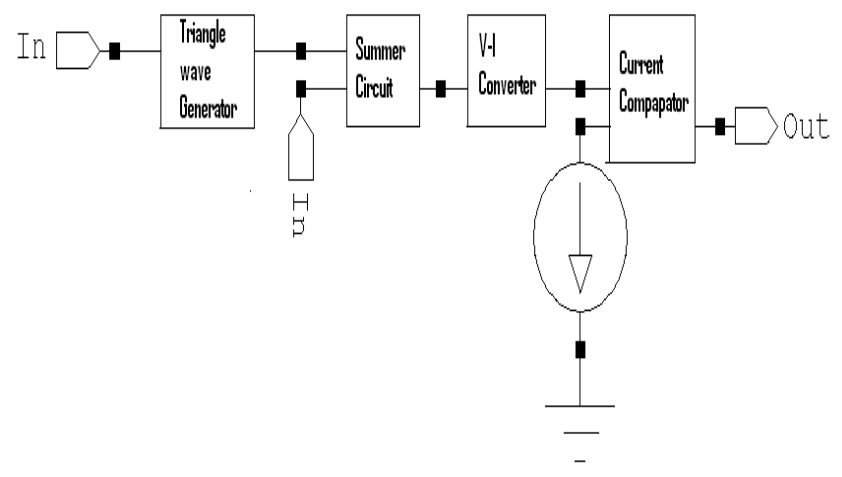

Fig.1 Block diagram of Pulse-width modulator

This paper is organized in four sections. Section I describes the principle of operation. And the scheme of PWM signals generation and signal forms are also described in this section. Section II shows the circuits description, which are used in proposed PWM. Section III shows the simulation results and section IV describes the conclusion respectively.

\section{PUlSE-WIdTh MOdUlation}

\section{Principle of operation}

Ordinarily the PWM signal can be classified into two forms [5]. One is PWM signal generated by natural sampling method which is called as natural sampling PWM signal (NPWM), another is PWM signal produced by uniform sampling method known as uniform sampling PWM signal (UPWM).For triangular carrier signal,it has two forms which the first is sawtooth signal known as 'single sided' signal because only a single edge of the PWM waveform is modulated.Another is triangular 
signal called 'double sided' signal $[6,7]$. For the proposed method NPWM signal will be introduced.

The proposed PWM is schematically depicted in Fig1. If we assumed that sine wave signal is modulating signal, and triangular wave is carrier signal. Then PWM output signal is generated by comparing the summation signal and reference current. We added a V-I converter after the summer circuit because we are using a current comparator to design the pulse-width modulator. We used a balanced low input impedance continuous-time CMOS current comparator for accurately performing pulse-width modulation.

\section{CirCUit IMPLEMENTATION}

\section{Current Comparator}

As we know, in general, the current comparator is applied to distinguish a current value from its reference value. However, we need to compare signals directly between two terminals with varied current in many applications. To perform direct comparison between two input currents, we have a balanced low input impedance continuous-time CMOS current comparator for accurately performing pulse-width modulation [8].

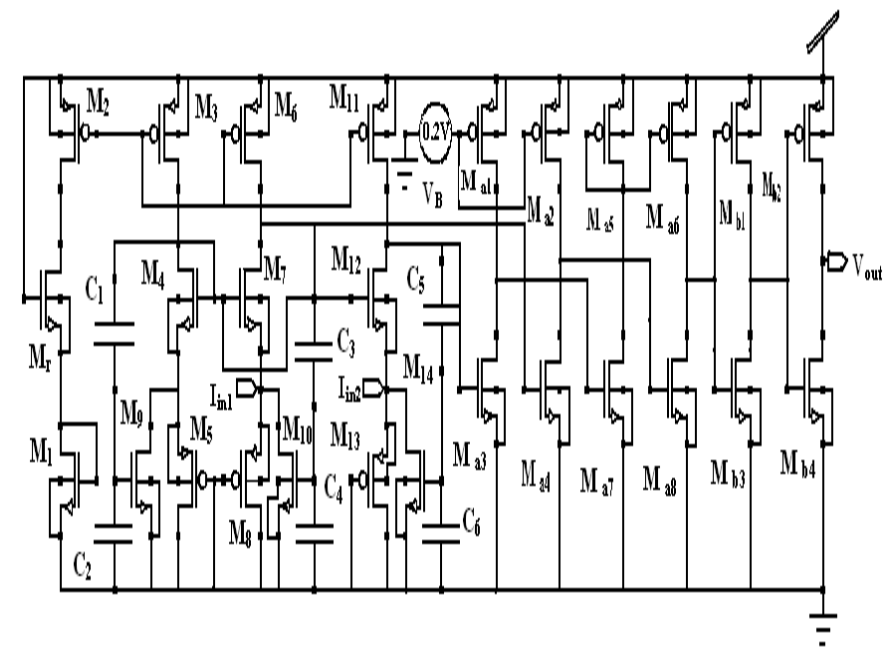

Fig.2 The schematic of current comparator.

The current comparator is applied to distinguish signals between two terminals with varied current in many applications [9]. For instance, in a multimedia system, we use comparator as a pulse-width modulator to modulate audio signal by a reference carrier signal. In this situation, the most significant consideration is the equilibrium of impedance between two input terminals. If the input
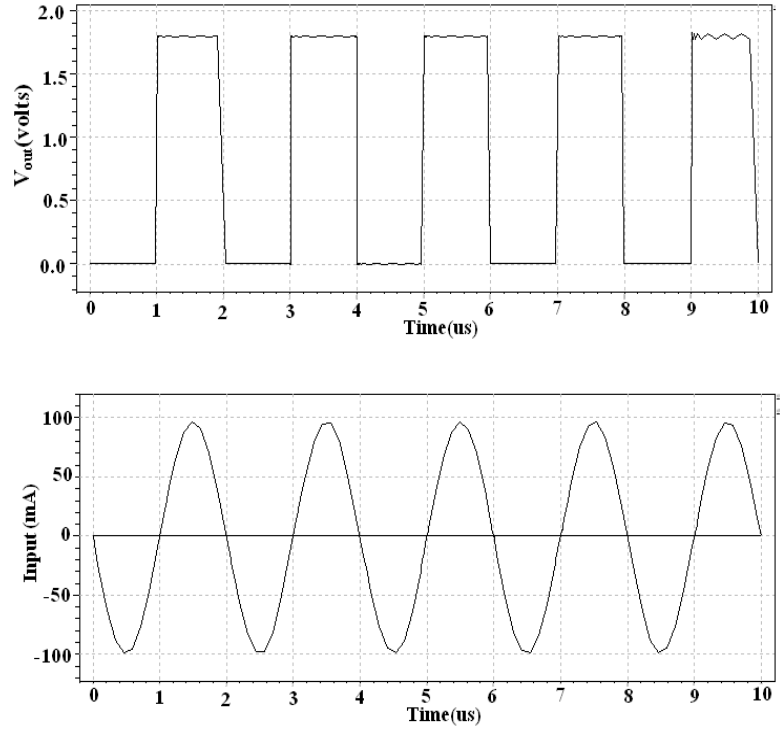

(a)
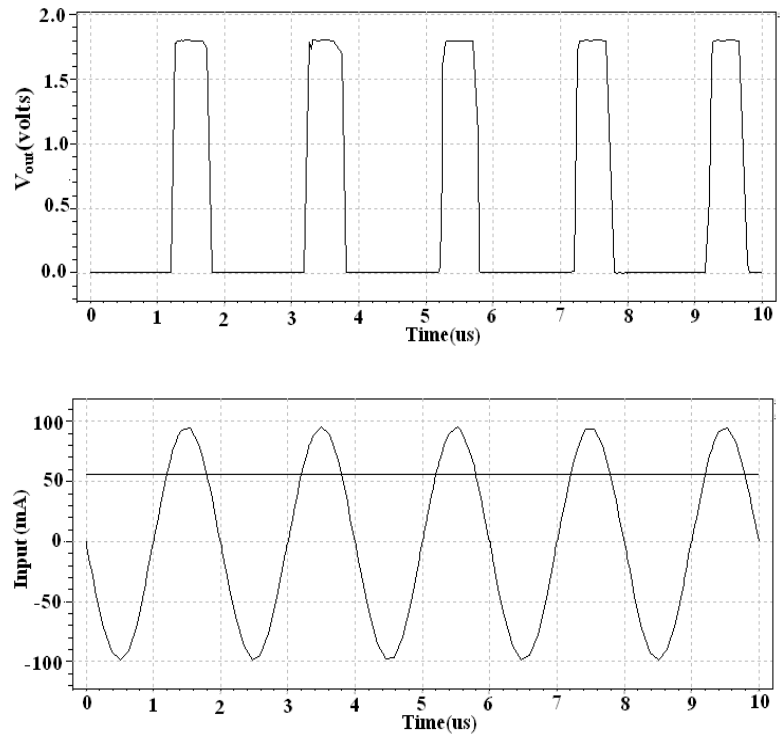

(b)

Fig.3 Transient waveforms for current comparator with input current of $100 \mathrm{~mA}$ and reference current is: (a) $0 \mathrm{~mA}$, (b) $60 \mathrm{~mA}$,

impedances are unbalanced, the validity of comparison would be mistrustful when the difference between two input signals is small. To properly perform direct comparison for two input signals, a current comparator shown in Fig.2. We add another parallel common-gate stage, $M_{11}$ to $M_{13}$, as a current buffer for the second input signal. Then, the common-source negative feedback loop consists of $\mathrm{C}_{5}, \mathrm{C}_{6}$ and $\mathrm{M}_{14}$ is similarly inserted to the common-gate input stage followed by a differential to single-end amplifier, $\mathrm{M}_{\mathrm{a} 8}$.

This equivalent input stages and feedback structures ideally provide balance and low input impedances. 
However, if we take process variation into consideration, the input impedances are not absolutely equivalent because the bias current from $\mathrm{M}_{6}$ is not entirely equal to that in $\mathrm{M}_{11}$ . We therefore have to put them in the same well during physical level implement to reduce the mismatch between these two input stages[10]. The transient waveform of current comparator is shown in Fig 3.

\section{Summer circuit:}

An operational amplifier can easily be made into a summing circuit by using additional input resistors. An analog adder or summer can be made using either an inverting amplifier or a non-inverting amplifier. However when using an inverting amplifier the output will be inverter, although still the sum of all the inputs. A summer circuit is shown below, which means that the output is the sum of the two inputs .

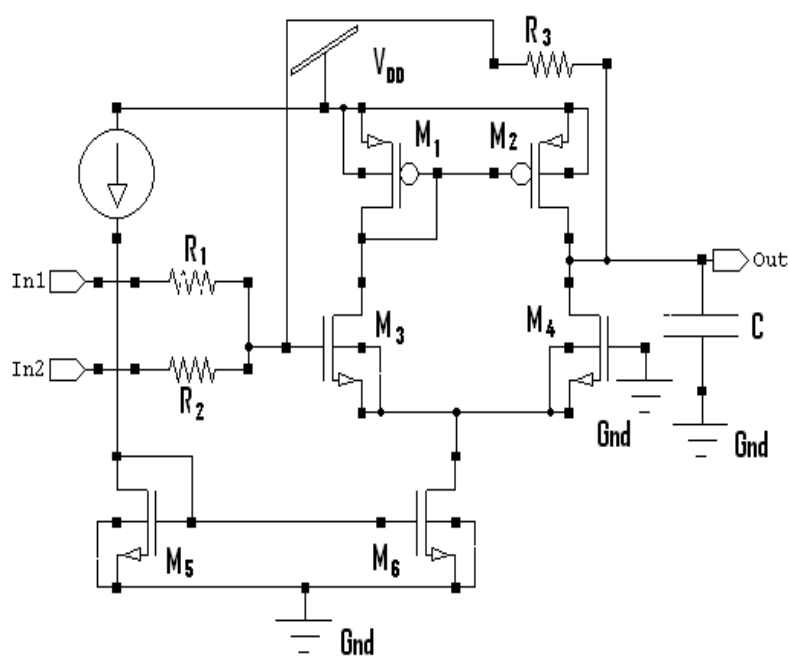

Fig.4 Schematic of summer circuit.
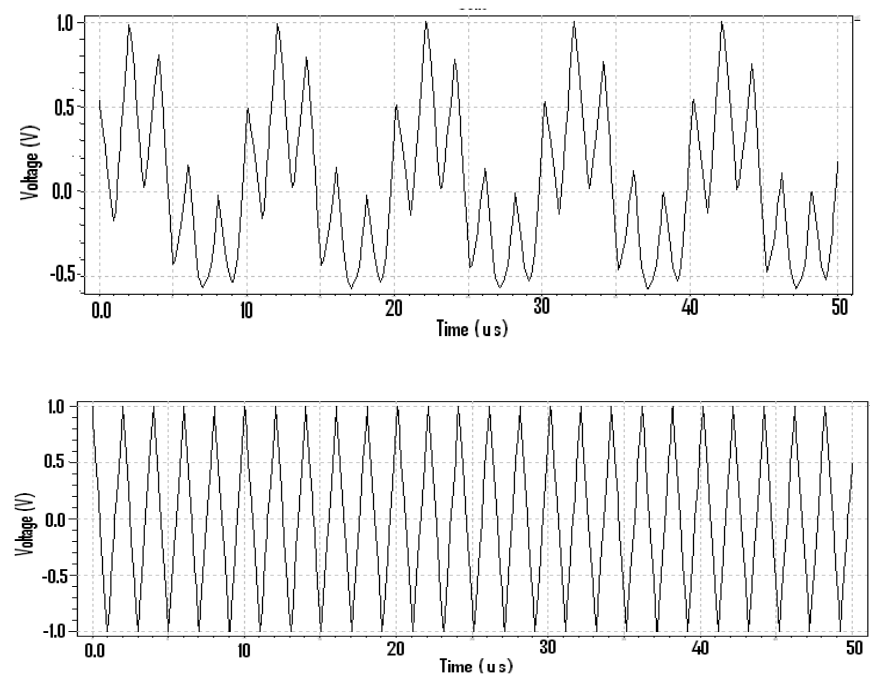

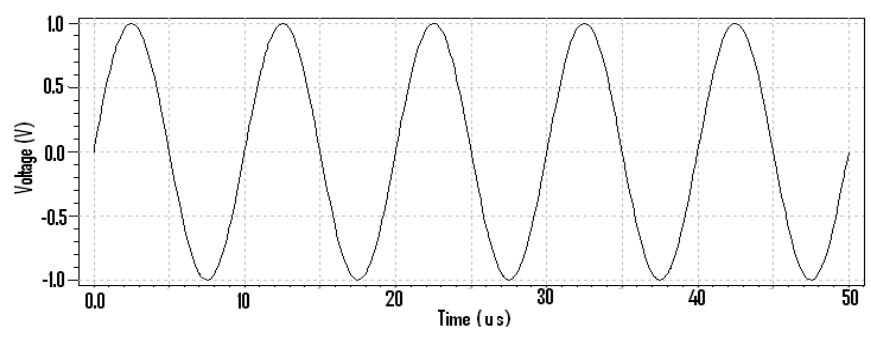

Fig.5.Transient waveforms of summer circuit

The two inputs are shown in the schematic, although any number of inputs over two could be used. The transient waveform of summer circuit are shown in Fig 5.

\section{Triangle wave generator:}

We know that the integrator output waveform will be triangular if the input to it is a square-wave. It means that a triangular-wave generator can be formed with an integrator by applying the square wave at the input of the integrator, as illustrated in Fig6. This circuit needs an op-amp, two capacitors, and two resistors. When the square wave is applied to the input of integrator, it drives the integrator which produces a triangular output

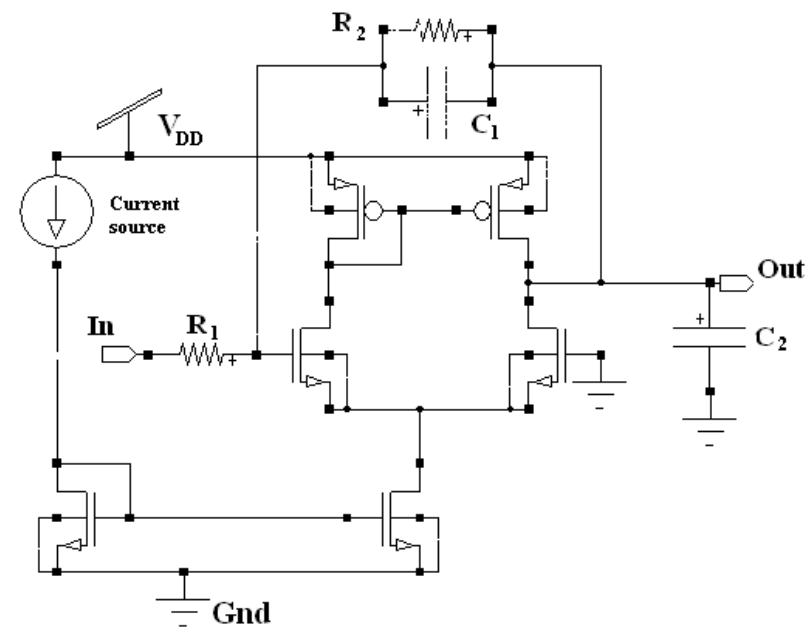

Fig.6 Schematic of Triangular wave generator.

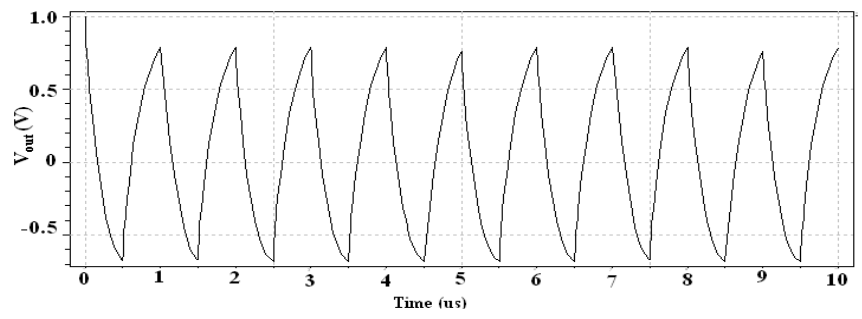




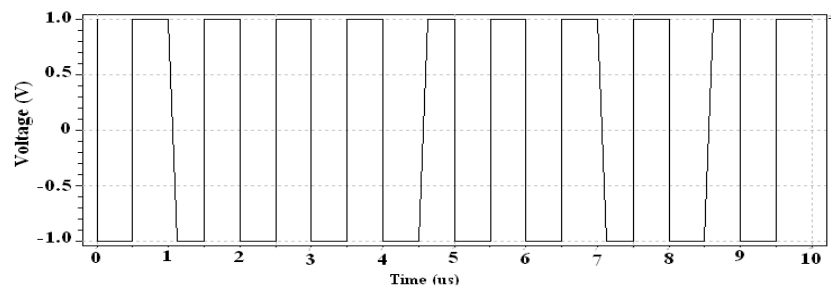

Fig.7 Transient waveforms of Triangular wave generator

waveform. The rectangular-wave swings between $+1 \mathrm{~V}$ and $-1 \mathrm{~V}$ is applied to the input. The triangular-waveform has the same period and frequency as the square-waveform. Peak to-peak value of output triangular-waveform can be obtained from the following equation[3].

The input of integrator is a square wave and its output is a triangular waveform, the output of integrator will be triangular wave only when $\mathrm{RC}>\mathrm{T} / 2$ where $\mathrm{T}$ is the period of square wave. As a general rule, $\mathrm{RC}$ should be equal to T. Since the frequency of the triangular-wave generator like any other oscillator, is limited by the op-amp slew-rate, a high slew rate op-amp, like LM 301, should be used for the generation of relatively higher frequency waveforms[4]. The transient waveform of triangular wave generator is shown in Fig7.

\section{Simulation Results}

In this section, we demonstrate the practicability of proposed design Pulse-width Modulator through implementing circuits of Fig. 1 by TSMC $0.35 \mu \mathrm{m}$ CMOS process. The experiment results are reported in Fig.8 to Fig 10 which shows the input and output waveform of Pulsewidth modulator and average power compared to that of prior work.
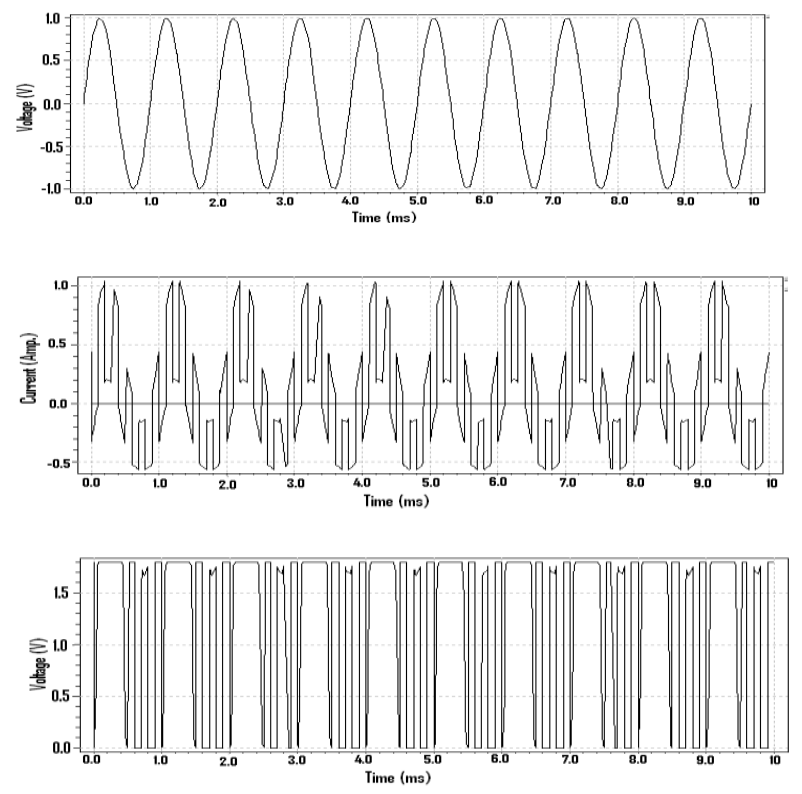

(a)
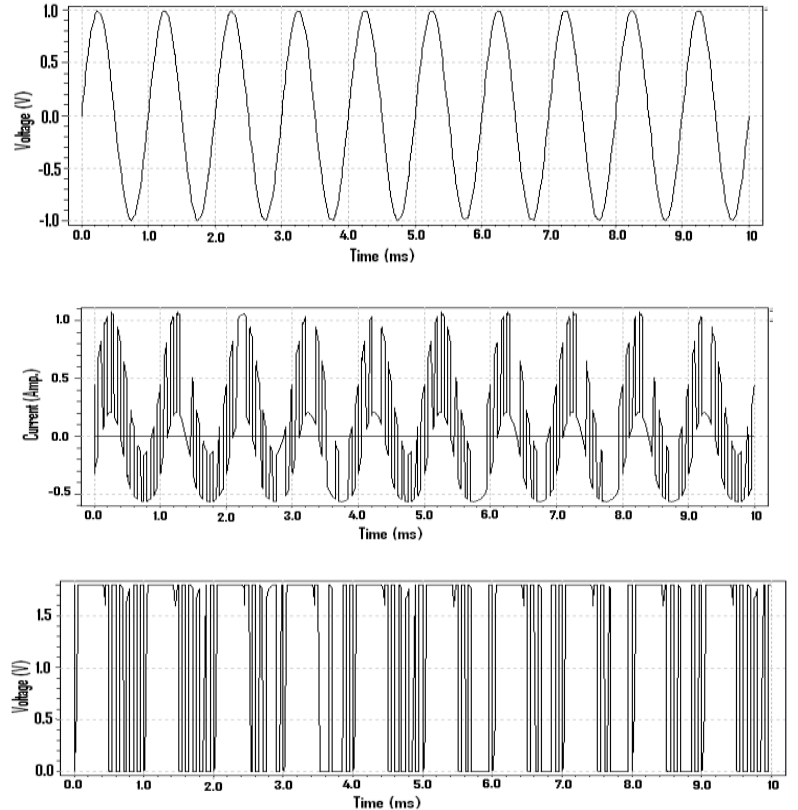

(b)
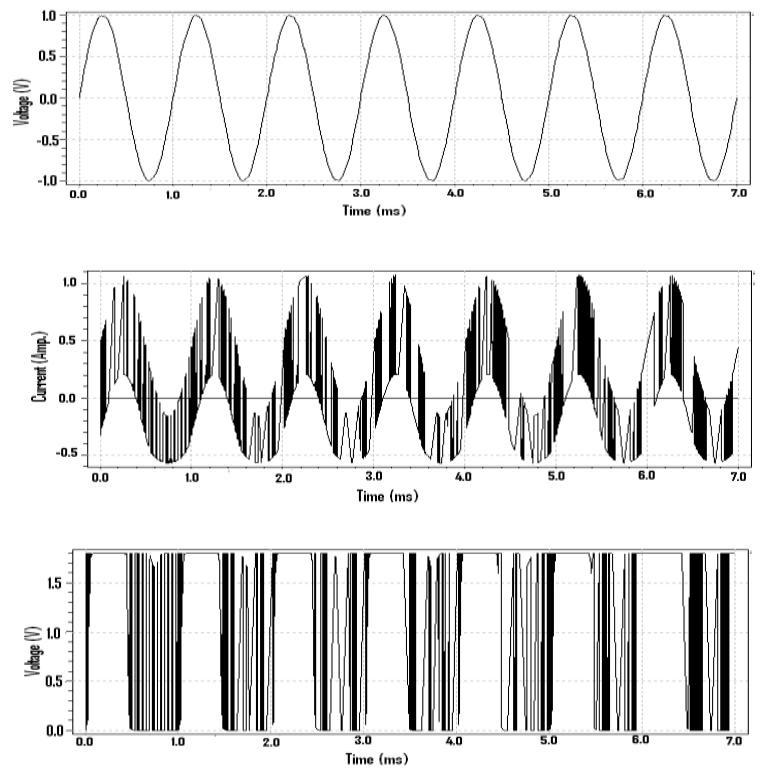

(c)
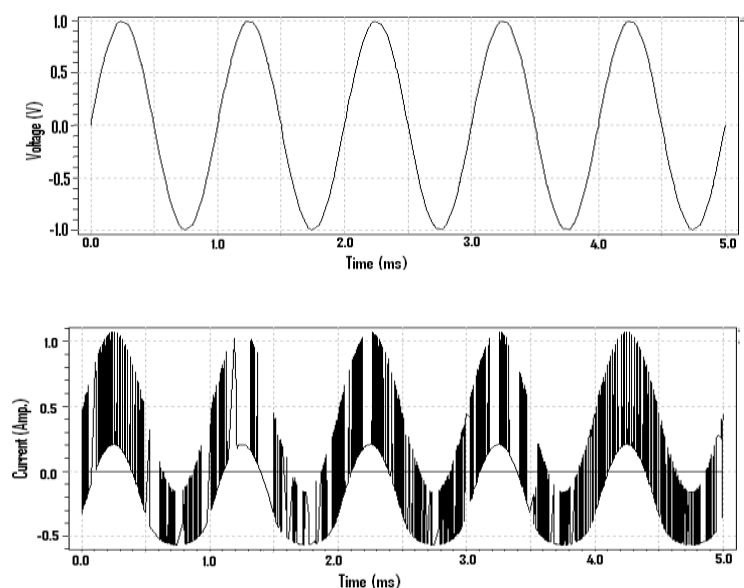


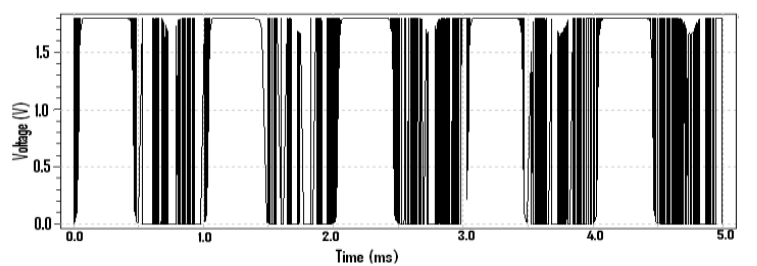

(d)

Figure.8.Transient waveforms of Pulse-width Modulator

Simulation result for the modulating signal of $1 \mathrm{kHz}$ and amplitude of $1 \mathrm{~V}(\mathrm{P}-\mathrm{P})$, reference level $0 \mathrm{~A}$ and carrier frequency as (a) $5 \mathrm{kHz}$ (b) $10 \mathrm{KHz}$ (c) $50 \mathrm{kHz}$ (d) 100 $\mathrm{KHz}$

Table.I Power consumed with different $V_{D D}$

\begin{tabular}{|c|c|}
\hline $\mathbf{V}_{\mathbf{D D}}($ Volts) & Power Consumed(watts) \\
\hline 1.6 & $1.474698 \mathrm{e}-003$ watts \\
\hline 1.7 & $1.784500 \mathrm{e}-003$ watts \\
\hline 1.8 & $2.141791 \mathrm{e}-003$ watts \\
\hline 1.9 & $2.550942 \mathrm{e}-003$ watts \\
\hline 2.0 & $3.001487 \mathrm{e}-003$ watts \\
\hline 2.1 & $3.498471 \mathrm{e}-003$ watts \\
\hline
\end{tabular}

Moreover, in Fig.8, we can see the transient waveform of the Pulse-width modulator for modulating signal of $1 \mathrm{kHz}$ and amplitude of $1 \mathrm{~V}$ peak to peak, reference level $0 \mathrm{~A}$ and carrier frequengy as $5,10,50,100 \mathrm{kHz}$ respectively. Fig 9 Shows the average power is of the proposed pulse -width modulator.

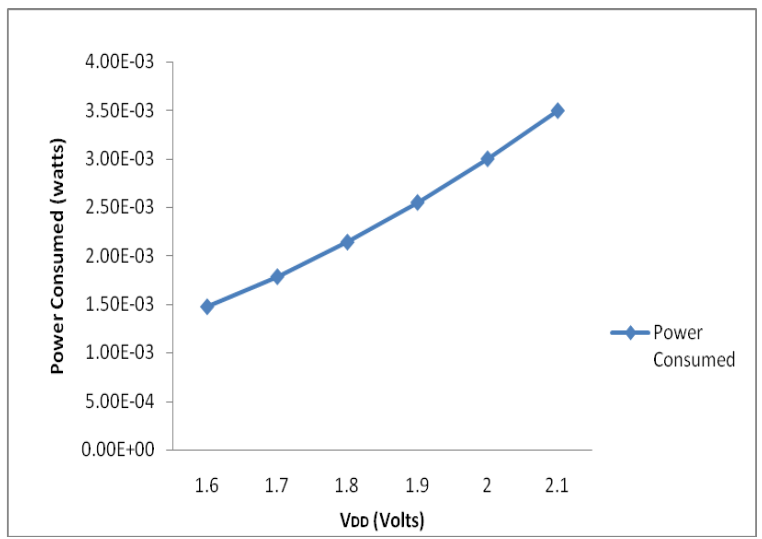

Fig.9 Power consumed with different $V_{D D}$

Table 1 shows the change in power consumed by the proposed Pulse-width Modulator with different $V_{D D}$. Fig 9 shows that the power consumed by the PWM circuit in increased linearly with increase in the power supply.

Table.II Power consumed with different input voltage

\begin{tabular}{|c|l|}
\hline $\mathbf{V}_{\text {in }}($ Volts $)$ & Power Consumed(watts) \\
\hline 0.5 & $2.129681 \mathrm{e}-003$ watts \\
\hline 0.6 & $2.132660 \mathrm{e}-003$ watts \\
\hline 0.7 & $2.136975 \mathrm{e}-003$ watts \\
\hline 0.8 & $2.141022 \mathrm{e}-003$ watts \\
\hline 0.9 & $2.141791 \mathrm{e}-003$ watts \\
\hline 1.0 & \\
\hline
\end{tabular}

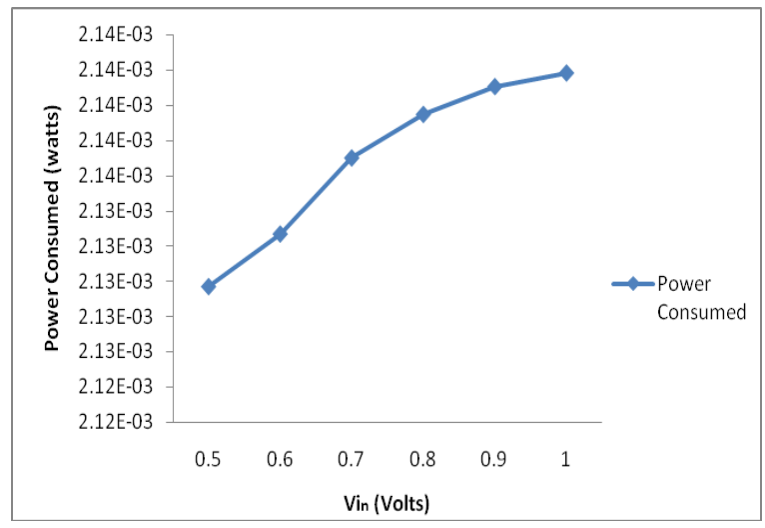

Fig.10. Power consumed with different Input voltage

Table 2 is the table of power consumed with different input voltage which is applied at the PWM input. The above graphs shown in Fig.10 is the graph of change in power consumed with different input voltage. This graph shows that the power consumed increase linearly with increase in input voltage.

\section{Conclusion}

In this paper, we have presented a simple Pulse-width Modulator. Pulse width modulation (PWM) is a powerful technique for controlling analog with a processors digital outputs. Experiment results show that the average Power consumed by the proposed Pulse-width modulator is about $3 \mathrm{~mW}$ under the implementation of TSMC $0.35 \mu \mathrm{m} \mathrm{CMOS}$ process with $1.8 \mathrm{~V}$ power supply. The current comparator used in the proposed pulse-width modulator can also compare the voltage input in place of current input. This advantage enables the current comparator to enhance the comparison accuracy while applying to pulse-width modulator. The summer circuit and triangular wave generator, used in the proposed PWM circuit, is designed with an operational amplifier in unity gain configuration. The proposed PWM is simpler than PWM IC form 
IOSR Journal of Engineering

Apr. 2012, Vol. 2(4) pp: 931-936

including it can yield the accurate PWM signal with widely operating carrier frequency. In addition the proposed scheme can be also realize in IC form

\section{ACKNOWLEDGEMENTS}

The authors would like to thank Mody Institute of Technology \& Science for supporting in carrying out this work.

\section{REFERENCES}

\section{Books:}

[1] Jerald G. Graeme, Application of operational Amplifiers third generation technique. Chapter 5. Mc-Graw hill Book company.1973.

[2] National semiconductor. " $O p$ Amp circuit collection." AN-31,1978.

[3] Kenneth R. Laker and Will,. M. C. Sansem"Design of Analog Integrated Circuits and Systems".McGraw Hill. iiic.. 1994.

[4] Plullip E. Allen and Douglas R. Holberg,"CMOS Analog Circuits Design". Saunders College Publishing, 1987.

\section{Proceedings Papers:}

[5] Goldberg J.M. and Sandler, M.B. "New high accuracy Pulse-width modulation based digital to analog converter/Power amplifier." IEEE Proc.Circuits Device Syst.141,4 (August 1994):315-324.

[6] Mellor, P.H.,Leigh, S.P. and B.M.G. Cheetham, "Reduction of Spectral distortion in Class D amplifiersby an enhanced Pulse-width modulation sampling Process." IEEE Prooceeding-G 138,4 (August 1991):441-448.

[7] Bresch , H., Streitenberger, M. and Mathis, W., "About the demodulation of PWM-signals with application to audio Amplifiers." Circuits and Systems, 1998. ISCAS '98.Proceeding of the 1998 IEEE International symposium. 1(1998):205208.

[8] H. Traff, "Novel approach to high speed CMOS current comparators," Electronics Letters, Vol.28, No.3, pp.310-312, 1992.

[9] X. Tang and K.-P. Pun, "High-performance CMOS current comparator," Electronics Letters, Vol.45, No.20, pp.1007-1009, 2009.

[10] Rockey Choudhary, Monika Bhardwaj, Prof.B.P Singh, Kopal Gupta "Design of low power low input Impedence current" in Second International Conference on Advanced Computing \& Communication Technologies, to be held on 7-8 Jan. 2012. 